\title{
Nonlinear Adaptive Blind Interference Cancellation for DS-CDMA Systems *
}

\author{
Dragan Samardzija \\ Wireless Communications Research Department, \\ Lucent Technologies, \\ 791 Holmdel-Keyport Road, Holmdel, NJ 07733, USA \\ dragan@lucent.com
}

\author{
Narayan Mandayam, Ivan Seskar \\ WINLAB, Rutgers University, \\ 73 Brett Road, Piscataway NJ 08854, USA \\ narayan, seskar@winlab.rutgers.edu
}

\begin{abstract}
In this paper we propose a multistage nonlinear blind interference cancellation (MS-NL-BIC) receiver for directsequence code-division multiple-access (DS-CDMA) systems. The receiver uses higher order statistics of the received baseband signal. Specifically, we use the second and fourth moments of the received signal to determine a component of the received vector that has significant mean energy and low variability of the energy which are favorable characteristics for application in an interference cancellation scheme that uses hard decisions. The structure of the receiver is multidimensional and can be viewed as a matrix of receivers. Each row in the matrix consists of receivers that perform (hard decisions) cancellation of successive components that have significant mean energy and low variability of the energy. The columns of the matrix essentially resemble multistage receivers that iteratively refine performance from earlier stages. Simulation results show that unlike linear receivers, the MS-NL-BIC is exceptionally efficient in systems with strong and highly correlated interferers, as may be the case in overloaded DS-CDMA systems.
\end{abstract}

\section{Introduction}

In DS-CDMA systems, in general, crosscorrelations between signature (spreading) sequences are nonzero. This results in multiple-access interference (MAI) which can disrupt reception of highly attenuated desired user signal. This is known as the near-far effect. To combat this problem several multiuser receivers have been proposed (for example, see $[3,5,6,1])$. These receivers are denoted as centralized because they require knowledge of parameters (signature sequences, amplitudes and timing) for all users in the system.

\footnotetext{
* This work is supported in part by the New Jersey Commission on Science and Technology under the New Jersey Center for Wireless Communication Technologies
}

Therefore, they are more suitable for processing at the base station.

For the downlink, it is desirable to devise decentralized receivers. Decentralized receivers exploit the knowledge of the desired user parameters only. The use of short signature sequences simplify the task of multiuser detection and interference cancellation, since a receiver can adaptively learn (estimate) the structure of the MAI [4]. Decentralized receivers may be further classified into data aided and nondata aided receivers. Unlike data aided receivers, blind (or nondata aided) multiuser detectors require no training data sequence, but only knowledge of the desired user signature sequence and its timing. The receivers treat MAI and background noise as a random process, whose statistics must be estimated. Majority of blind multiuser detectors are based on estimation of second order statistics of the received signal. In [2], a blind adaptive MMSE multiuser detector is introduced (proven to be equivalent to the minimum output energy (MOE) detector). A subspace approach for blind multiuser detection is presented in [13]; where both the decorrelating and the MMSE detector are obtained blindly. Further, adaptive and blind solutions are analyzed in [11], with an overview in [4]. A blind successive interference cancellation (SIC) scheme, which uses second order statistics, is proposed in [8]. A comprehensive treatment of multiuser detection can be found in [12].

In this paper we propose a novel blind interference cancellation receiver, which assumes knowledge of only the desired user's signature sequence. The receiver is based on determining that component of the received signal that has significant mean energy and low variability in the energy. It applies the minimum variance of energy and maximum mean energy criterion (MVE-MME), which is described in Section 3. In Section 4, using the above criterion, we derive a nonlinear multistage blind interference cancellation (NL-MS-BIC) receiver. The structure of the NL-MS-BIC receiver is multidimensional and can be viewed as a matrix of IC stages. Each row in the matrix consists of IC stages that perform the blind (hard decisions) successive interfer- 
ence cancellation. The columns of the matrix essentially resemble multistage receivers that iteratively refine performance from earlier stages. Simulation results are presented in Section 5, and we conclude in Section 6.

\section{Background}

We now present the asynchronous DS-CDMA system model. The received baseband signal, $r(t)$, in a $K$-user asynchronous DS-CDMA additive white Gaussian noise (AWGN) system is

$$
r(t)=\sum_{i=-J}^{J} \sum_{k=1}^{K} A_{k} b_{k}(i) s_{k}\left(t-i T-\tau_{k}\right)+\sigma n(t)
$$

where $A_{k}$ is the received amplitude, $b_{k}(i) \in\{-1,+1\}$ is binary, independent and equiprobable data, $s_{k}(t)$ is the signature sequence which is assumed to have unit energy, $\tau_{k}$ is the relative time offset, all for the $k^{t h}$ user. $T$ is the symbol period and $n(t)$ is AWGN with unit power spectral density, with $\sigma$ being square root of the noise power. $2 J+1$ is the number of data symbols per user per frame.

It is well known that an asynchronous system with independent users can be analyzed as synchronous if equivalent synchronous users are introduced, which are effectively additional interferers [12]. In this paper we consider the received signal $r(t)$ over only one symbol period that is synchronous to the desired user $(k=1)$. The discrete representation for the received signal in (1) can be written in vector form as

$$
\mathbf{r}=\sum_{k=1}^{L} A_{k} b_{k} \mathbf{s}_{k}+\sigma \mathbf{n}
$$

where the number of the interferers $(L-1=2(K-1))$ is doubled due to the equivalent synchronous user analysis. $\mathbf{r}, \mathbf{s}_{k}$ and $\mathbf{n}$ are vectors in $\Re^{M}$, where $M$ is the number of chips per bit.

Let us now repeat the outline of the nonlinear centralized SIC scheme which is presented in [6,9]. We present this scheme because its approach to nonlinear interference cancellation is generalized and later applied in a blind interference cancellation scheme (Section 4). In the nonlinear centralized SIC scheme it is assumed that the signature sequences are perfectly known (centralized approach). The basic operations of the SIC algorithm are (see Figure 1):

1. Detect one user with the conventional detector, i.e., matched filter (MF).

2. Regenerate the baseband signal (vector) for this user.

3. Cancel the regenerated signal (vector) from the received baseband signal.

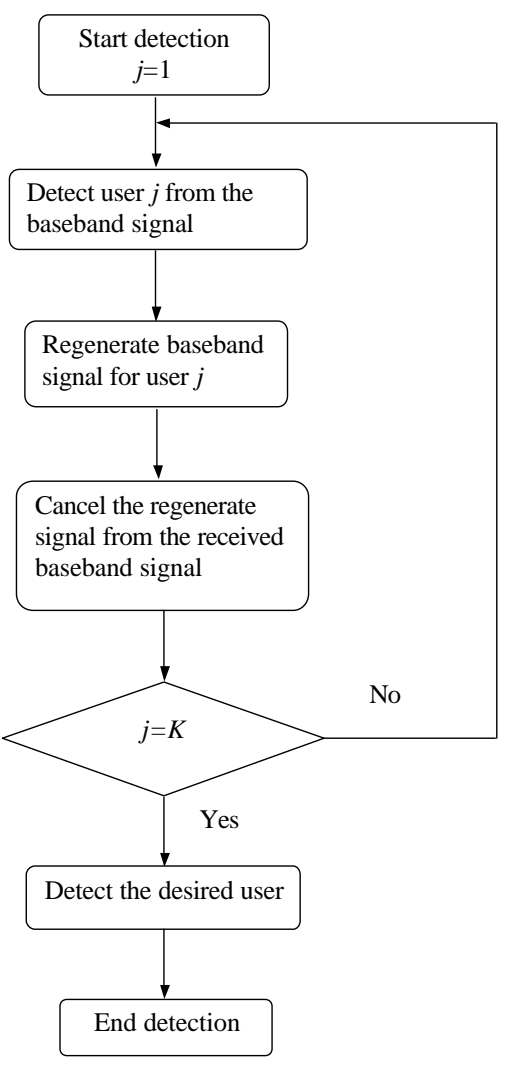

\section{Figure 1. Flow chart illustrating the nonlinear centralized SIC scheme.}

Then, this operation is repeated successively for all the users in the system. The idea is that successive cancellations result in reduced MAI for the remaining users. The received vector $\mathbf{r}_{j+1}$ after stage $j$ of the cancellation is given by

$$
\mathbf{r}_{j+1}=\mathbf{r}_{j}-\hat{A}_{j} \hat{b}_{j} \mathbf{s}_{j}
$$

where $\mathbf{r}_{j}$ is the received vector, $\hat{A}_{j}$ and $\hat{b}_{j}$ are the corresponding estimates of the amplitude and the bit, respectively, all for stage $j$. The above implementation of the SIC algorithm is nonlinear in that it uses hard decisions $\left(\hat{b}_{j} \in\right.$ $\{-1,1\})$ in successive stages. A primary reason why the nonlinear centralized SIC cannot achieve performance of the single user lower bound (SULB) is due to erroneous estimate of bit decision (i.e., $\hat{b}_{j} \neq b_{j}$ ). When an error happens, it causes the SIC scheme to double the interference, which is, of course, undesirable. Furthermore, the doubled interference propagates through the following IC stages, which degrades the overall performance of the receiver. For the same reason, this receiver also is also not near-far resistant [12]. Further, imperfections in amplitude and delay estimates can lead to the non ideal regeneration and cancellation. 
Accordingly, to obtain best results, the user with the highest signal to interference ratio (SIR) should be cancelled first. This condition is usually relaxed and the user with highest received power is cancelled first, followed by the second strongest, and so forth $[6,1]$. Thus, it is desirable to identify users (or signature sequences) that have significant power (energy). Note that the SIC scheme requires amplitude estimates for the users, which implicitly requires low variability of the amplitude estimates for perfect cancellation.

Let us now generalize the nonlinear cancellation given by (3). In equation (3), let us replace $\mathbf{s}_{j}$ with a vector $\mathbf{v}_{j}$ (not necessarily a signature sequence). Furthermore, we replace $\hat{A}_{j}$ with the square root of the estimate of $\beta_{j}=E\left[\left(\mathbf{r}_{j}^{\top} \mathbf{v}_{j}\right)^{2}\right]$, and $\hat{b}_{j}$ with $\operatorname{sgn}\left(\mathbf{r}_{j}^{\top} \mathbf{v}_{j}\right)$. Thus, the nonlinear cancellation in the $j^{\text {th }}$ stage is executed as

$$
\mathbf{r}_{j+1}=\mathbf{r}_{j}-\sqrt{\hat{\beta}_{j}} \operatorname{sgn}\left(\mathbf{r}_{j}^{\top} \mathbf{v}_{j}\right) \mathbf{v}_{j}
$$

In the following, we propose favorable characteristics of the vector $\mathbf{v}_{j}$ to be successfully applied in the above scheme. We now analyze the estimate of the energy $\beta_{j}$ of the received signal in the direction of the vector $\mathbf{v}_{j}$. To estimate $\beta_{j}$ we use sample statistics as

$$
\hat{\beta}_{j}(n)=\frac{1}{N} \sum_{m=n-N+1}^{n}\left(\mathbf{r}^{\top}(m) \mathbf{v}_{j}\right)^{2}
$$

where $N$ is the size of the averaging window (number of samples), and $n$ and $m$ are time indices (will be omitted in the following). It is well known that the error of the estimate in (5) is directly related to the variance of $x_{j}=\left(\mathbf{r}^{\top} \mathbf{v}_{j}\right)^{2}$. Using the Chebyshev inequality [7] it can be shown that as the variance of $x_{j}$ gets lower, the accuracy (mean squared error) of the energy estimate is improved:

$$
E\left[\left(x_{j}-E\left[x_{j}\right]\right)^{2}\right] \downarrow \Rightarrow E\left[\left(\hat{\beta}_{j}-\beta_{j}\right)^{2}\right] \downarrow
$$

We can say that for the vector $\mathbf{v}_{j}$ that corresponds to $x_{j}$ with lowest variance (among all vectors in $\Re^{M}$ ), the estimate of the energy is the most reliable, i.e., the mean squared error of the energy estimate is the lowest. Note that the variance of $x_{j}$ is the variance of the energy of the received vector $\mathbf{r}$ in the direction of the vector $\mathbf{v}_{j}$ (i.e., variance of squared projection of the vector $\mathbf{r}$ onto the vector $\mathbf{v}_{j}$ ).

The above analysis leads us to believe that the vector $\mathbf{v}_{j}$ which corresponds to the low variability of the energy and significant mean energy of the vector $\mathbf{r}$ in the direction of $\mathbf{v}_{j}$, is desirable for the nonlinear cancellation given by (4). These characteristics of the vector $\mathbf{v}_{j}$ offer reliable estimates of the corresponding energy $\beta_{j}$ and sign of $\mathbf{r}^{\top} \mathbf{v}_{j}$. In the following we present a scheme that blindly determines (estimates) the vector $\mathbf{v}_{j}$ and further applies this vector to realize a multistage nonlinear interference cancellation scheme.

\section{MVE-MME Optimization Criterion}

We now present an optimization criterion which is used in deriving a nonlinear blind adaptive interference cancellation scheme. According to the analysis in Section 2, the goal of the optimization approach is to determine a component of the received vector $\mathbf{r}$ that has low variability in the energy and significant mean energy. We consider the squared output of the projection of $\mathbf{r}$ onto a vector $\mathbf{v} \in \Re^{M}$. The vector $\mathbf{v}$ is obtained from the following nonlinear procedure which is

$$
\mathbf{v}=\arg \min _{\mathbf{u}}\left\{\alpha(\mathbf{u})=(1-\mu) \alpha_{1}(\mathbf{u})-\mu \alpha_{2}(\mathbf{u})\right\}
$$

where $\mathbf{u} \in \Re^{M}$ is subject to $\mathbf{u}^{\top} \mathbf{u}=1$ and $0<\mu<1$. The function $\alpha_{1}(\mathbf{u})$ denotes the variance of the squared output $\mathbf{r}^{\top} \mathbf{u}$ and is given as

$$
\alpha_{1}(\mathbf{u})=E\left[\left(\left(\mathbf{r}^{\top} \mathbf{u}\right)^{2}-E\left[\left(\mathbf{r}^{\top} \mathbf{u}\right)^{2}\right]\right)^{2}\right]
$$

The function $\alpha_{2}(\mathbf{u})$ in (7) denotes the mean squared energy given as

$$
\alpha_{2}(\mathbf{u})=E\left[\left(\mathbf{r}^{\top} \mathbf{u}\right)^{4}\right]
$$

We now present the following proposition that gives an intuitive description of the minimum variance of energy criterion, which is obtained by minimizing the expression in (8).

Proposition 1 For the synchronous antipodal DS-CDMA system (described in (2)), with zero AWGN $(\sigma=0)$ and linearly independent signature sequences $\left\{\mathbf{s}_{i}\right\}_{i=1}^{L}$, the solutions for $\mathbf{w}=\arg \min _{\mathbf{u}}\left(\alpha_{1}(\mathbf{u})\right)$, constrained as $\mathbf{u}^{\top} \mathbf{u}=1$, are classified in two groups:

(a) $\mathbf{w}= \pm \mathbf{d}_{i}(i=1, \cdots, L)$, where $\mathbf{d}_{i} \perp \operatorname{span}\left\{\mathbf{s}_{k}\right\}_{k=1, k \neq i}^{L}$ and $\mathbf{s}_{i}^{\top} \mathbf{d}_{i} \neq 0$. Actually, $\mathbf{d}_{i}$ is the modified matched filter that corresponds to decorrelating detector for user $i$ [3].

(b) $\mathrm{w}$ is any vector from the noise subspace.

Further, the above solutions correspond to the absolute minimum of $\alpha_{1}(\mathbf{u})$ where it reaches zero.

Further, it can be shown that the vector $\mathbf{v}_{\max }=$ $\arg \max _{\mathbf{u}}\left(\alpha_{2}(\mathbf{u})\right)$, constrained as $\mathbf{u}^{\top} \mathbf{u}=1$, is equal to the vector that also maximizes the mean energy $E\left[\left(\mathbf{r}^{\top} \mathbf{u}\right)^{2}\right]$. It is shown in [8] that $\mathbf{v}_{\max }$ is the eigenvector that corresponds to the largest eigenvalue of the input covariance matrix $\mathbf{R}_{r}=E\left[\mathbf{r} \mathbf{r}^{\top}\right]$. Instead of the mean energy $E\left[\left(\mathbf{r}^{\top} \mathbf{u}\right)^{2}\right]$ (second order statistics), $E\left[\left(\mathbf{r}^{\top} \mathbf{u}\right)^{4}\right]$ is applied in (7) such that both terms $\left(\alpha_{1}()\right.$ and $\left.\alpha_{2}()\right)$ are statistics of the same order (i.e., fourth order statistics). Based on the above, the vector $\mathbf{v}$ corresponds to that component of the received signal $\mathbf{r}$ that has low variability in the energy and significant mean energy. These characteristics are favorable for application of the vector $\mathbf{v}$ in a nonlinear interference cancellation scheme (see Section 2). The parameter $\mu$ is used to control which of these two characteristics (low variability of the 
energy or significant mean energy) is dominant. For example, if $\mu=0$, the optimization in (7) is equivalent to minimum variance of energy (MVE), and for $\mu=1$ it is equivalent to maximum mean energy (MME) optimization criterion [8]. Therefore, we refer to (7) as the minimum variance of energy and maximum mean energy (MVE-MME) optimization criterion.

We now present an adaptive algorithm that solves (7). We exploit some properties of the functions given in (8) and (9). Let us assume that the input process $\mathbf{r}$ is wide sense stationary (WSS) and also that

$$
\begin{gathered}
E\left[\left(\mathbf{r}^{\top}(n) \mathbf{u}\right)^{2}\left(\mathbf{r}^{\top}(n+m) \mathbf{u}\right)^{2}\right]= \\
=E\left[\left(\mathbf{r}^{\top}(n) \mathbf{u}\right)^{2}\right] E\left[\left(\mathbf{r}^{\top}(n+m) \mathbf{u}\right)^{2}\right]
\end{gathered}
$$

where $n$ and $m$ are time indices, and $n \neq m$. In other words, we assume that the energy of $\mathbf{r}$ in direction of the vector $\mathbf{u}$ is uncorrelated in different symbol (bit) intervals. Using the properties of WSS processes and (10) we can show that (8) can be written as

$$
\alpha_{1}(\mathbf{u})=\frac{1}{2} E\left[\left(\left(\mathbf{r}^{\top}(n) \mathbf{u}\right)^{2}-\left(\mathbf{r}^{\top}(n+m) \mathbf{u}\right)^{2}\right)^{2}\right]
$$

for all integer $n$ and $m, n \neq m$. Similarly, the expression (9) can be written as

$$
\alpha_{2}(\mathbf{u})=\frac{1}{2} E\left[\left(\mathbf{r}^{\top}(n) \mathbf{u}\right)^{4}+\left(\mathbf{r}^{\top}(n+m) \mathbf{u}\right)^{4}\right]
$$

According to (11) and (12), and using sample statistics, the function $f(\mathbf{u})$ is defined as an approximation of $\alpha(\mathbf{u})$ as

$$
\begin{aligned}
f(\mathbf{u}, n)= & \frac{1}{2} \sum_{m=1}^{F}\left[(1-\mu)\left(\left(\mathbf{r}^{\top}(n) \mathbf{u}\right)^{2}-\left(\mathbf{r}^{\top}(n+m) \mathbf{u}\right)^{2}\right)^{2}-\right. \\
& \left.-\mu\left(\left(\mathbf{r}^{\top}(n) \mathbf{u}\right)^{4}+\left(\mathbf{r}^{\top}(n+m) \mathbf{u}\right)^{4}\right)\right]
\end{aligned}
$$

The time index $n$ is omitted in the following. $F$ is the number of consecutive symbols used for the approximation. Let us now consider a stochastic gradient algorithm [10] that solves (7) as

$$
\hat{\mathbf{v}}^{l+1}=\hat{\mathbf{v}}^{l}-\gamma \nabla\left(f\left(\hat{\mathbf{v}}^{l}\right)\right)
$$

where $l$ is the index of the iteration step, and $0<\gamma<1$ is a certain scalar which defines the length of adaptation step. The constraint $\left|\hat{\mathbf{v}}^{l+1}\right|=1$ is forced after every iteration where $\hat{\mathbf{v}}^{l}$ stands for estimate of $\mathbf{v}$ in $l^{t h}$ iteration step.

\section{Application of the MVE-MME Criterion in the Multistage Nonlinear Blind IC Receiver}

We now present the multistage nonlinear blind interference canceler, denoted as MS-NL-BIC. The structure of the receiver is multidimensional and can be viewed as a matrix of receivers (i.e., matrix of IC stages). The MS-NL-BIC receiver consists of $P$ rows and $Q$ columns, where each entry of the matrix corresponds to an interference cancellation stage denoted as $\mathbf{I C}_{i j}(i=1, \ldots, P, j=1, \ldots, Q)$. The following steps are executed in the $\mathbf{I C}_{i j}$ stage (where $\mathbf{r}_{i j}$ is the input vector to that stage):

1. Add back $\mathbf{x}_{(i-1) j}$ as

$$
\mathbf{r}_{i j}^{\prime}=\mathbf{r}_{i j}+\mathbf{x}_{(i-1) j}
$$

where $\mathbf{x}_{(i-1) j}$ is a portion of the received signal that is cancelled in the $\mathbf{I} \mathbf{C}_{i-1 j}$ stage. Note that the $\mathbf{I} \mathbf{C}_{i-1 j}$ stage is the same column, but earlier row of the matrix. For the first row $(i=1), \mathbf{x}_{0 j}=0(j=1, \cdots, Q)$ and $\mathbf{r}_{11}=\mathbf{r}$, because no cancellation is performed prior to this row.

2. Use adaptation rule in (14) $\left(\mathbf{r}_{i j}^{\prime}\right.$ replaces $\left.\mathbf{r}\right)$ to estimate $\mathbf{v}_{i j}$ as $\hat{\mathbf{v}}_{i j}$ (see Figure 2). Note that the vector $\hat{\mathbf{v}}_{i j}$ is further processed in the very same manner as an interferer signature sequence in the case of the nonlinear centralized SIC scheme (see Section 2).

3. Estimate the energy $\beta_{i j}=E\left[\left(\mathbf{r}_{i j}^{\prime}{ }^{\top} \hat{\mathbf{v}}_{i j}\right)^{2}\right]$. Note that the estimation should be reliable because $\hat{\mathbf{v}}_{i j}$, as a component of the vector $\mathbf{r}_{i j}^{\prime}$, has low variability in the energy (due to the term $(1-\mu) \alpha_{1}(\mathbf{u})$ in (7)).

4. Detect the sign of $\mathbf{r}_{i j}^{\prime}{ }^{\top} \hat{\mathbf{v}}_{i j}$. Note that detection should be reliable, because the component $\hat{\mathbf{v}}_{i j}$ has significant mean energy (due to the term $-\mu \alpha_{2}(\mathbf{u})$ in (7)) and low variability.

5. Perform nonlinear cancellation as

$$
\mathbf{r}_{i j+1}=\mathbf{r}_{i j}^{\prime}-\mathbf{x}_{i j}
$$

where (see Figure 3)

$$
\mathbf{x}_{i j}=\operatorname{sgn}\left(\mathbf{r}_{i j}^{\prime}{ }^{\top} \hat{\mathbf{v}}_{i j}\right) \sqrt{\hat{\beta}_{i j}} \hat{\mathbf{v}}_{i j}
$$

The above procedure is executed successively (within the $i^{\text {th }}$ row of the matrix), where for the new stage $\mathbf{I} \mathbf{C}_{i j+1}$, the input vector is $\mathbf{r}_{i j+1}$ (see equation (16)). The structure of the $i^{\text {th }}$ row (i.e., horizontal topology) is depicted in Figure 4. From the above, each row may be viewed as a blind equivalent to the nonlinear centralized SIC scheme, where the components $\hat{\mathbf{v}}_{i j}$ replace the actual signature sequence. After sufficient number $Q$ of the stages in the $i^{\text {th }}$ row, cancellation is repeated in the $(i+1)^{t h}$ row (see Figure 5). In Section 5, $Q$ is equal to the number of dominant interferers. The input vector $\mathbf{r}_{i+11}$ of the $(i+1)^{t h}$ row is $\mathbf{r}_{i Q+1}$. The stage $\mathbf{I} \mathbf{C}_{i+1 j}$ is used to iteratively refine the cancellation which is executed 


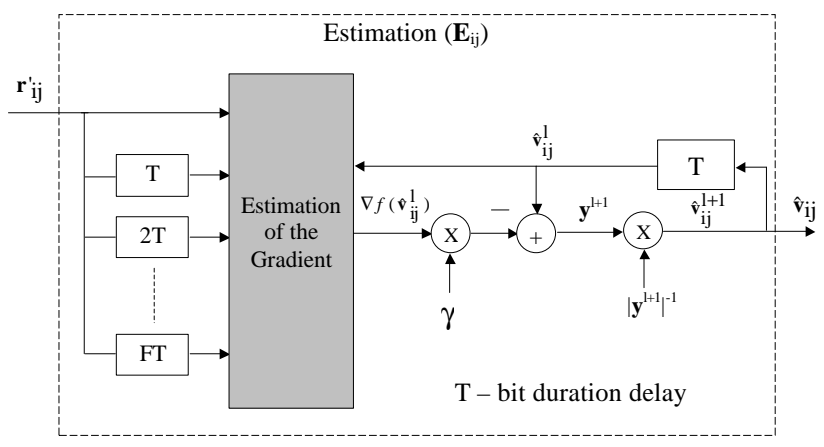

Figure 2. Block scheme: estimation of the vector $\mathbf{v}_{i j}$.

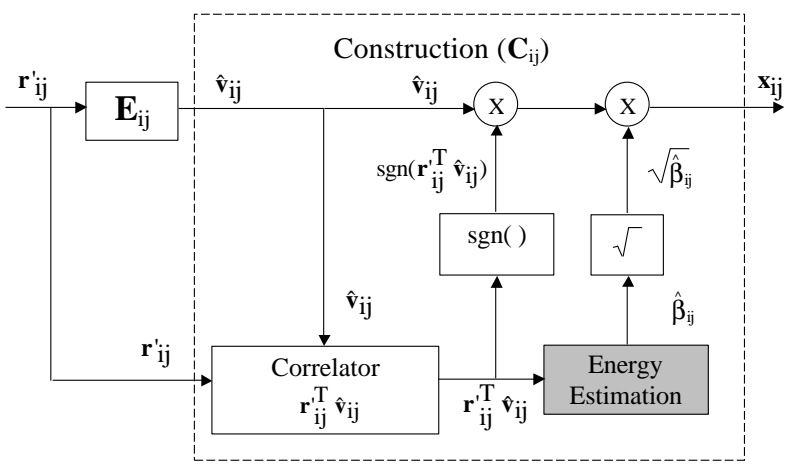

Figure 3. Block scheme: construction of the vector $\mathrm{x}_{i j}$.

in the earlier stage $\mathbf{I C}_{i j}(j=1, \ldots, Q)$. With appropriate delay, the vector $\mathbf{x}_{i j}$, that is canceled in the stage $\mathbf{I} \mathbf{C}_{i j}$ is added back (step 1), and within the stage $\mathbf{I C}_{i+1 j}$ processing is performed again (steps 2 to 5 ). The number of the rows $P$ is directly related to the performance of the receiver. Thus, the trade-off in performance versus complexity can be controlled by the number $P$. After sufficient number $P$ of the rows, detection of the desired user is performed using a linear detector (e.g., matched filter). Note that implicit in the above algorithm is the assumption that the interferers are stronger than the desired user. If the desired user is stronger than the interferers, then additional processing is required to ensure that the desired signal is not canceled out before the detection. In the following we assume that the interferers are stronger than the desired user.

\section{Simulation Results}

We consider a synchronous AWGN DS-CDMA system using randomly generated signature sequences with processing gain $M=8$. The users are independent and the following cases are analyzed:

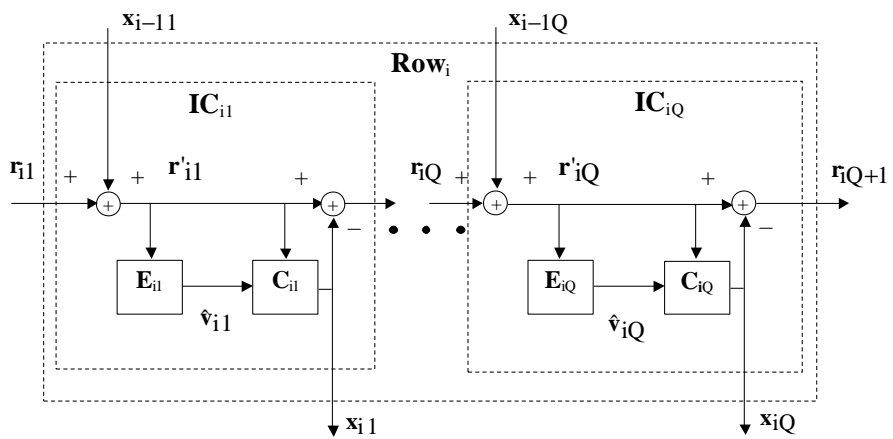

Figure 4. Horizontal topology of the MS-NLBIC receiver.

1. System with $L=8$ users (fully loaded), and equalenergy interferers: $A_{i}^{2} / A_{1}^{2}=25, i=2, \cdots, 8$.

2. System with $L=4$ users, and equal-energy interferers: $A_{i}^{2} / A_{1}^{2}=25, i=2, \cdots, 4$.

3. System with $L=12$ users (overloaded system); three strong equal-energy interferers: $A_{i}^{2} / A_{1}^{2}=25, i=$ $2, \cdots, 4$, and eight interferers with the same energy as the desired user: $A_{i}^{2} / A_{1}^{2}=1, i=5, \cdots, 12$.

The crosscorrelation profile of the users with respect to the desired user is depicted in Figure 6. Note that in this particular example the crosscorrelations are very high, except for users 5,10 and 12 which happen to be orthogonal to the desired user. In the case 1 , the system has users $i=1, \cdots, 8$, and in the case 2 only $i=1, \cdots, 4$. In all our results the input sample covariance matrix is estimated according to

$$
\hat{\mathbf{R}}_{r}(i)=\frac{1}{N} \sum_{k=i-N+1}^{i} \mathbf{r}(k) \mathbf{r}^{\top}(k)
$$

where $N$ is the size of the averaging window (number of samples), and $i$ is the time index (will be omitted in the following text). Performance of the conventional matched filter (MF), the centralized MMSE receiver (denoted as MMSE), the blind MMSE receiver (BMMSE) (detector $\hat{\mathbf{c}}=$ $\hat{\mathbf{R}}_{r}^{-1} \mathbf{S}_{\mathbf{1}}$ [13]) and the single user lower bound (SULB) are used as benchmarks for evaluation of the MS-NL-BIC receiver. The centralized MMSE assumes perfect knowledge of all the signature sequences, amplitudes and the variance of the AWGN. Performance of the MS-NL-BIC is evaluated for MF (MS-NL-BIC-(MF)) and the blind MMSE (MSNL-BIC-(MMSE)); where these linear detectors are used for detection of the desired user after the cancellation (after $P$ rows and $Q$ IC stages within each row). The MS-NL-BIC(MMSE) uses the sample covariance matrix of the output signal of the last IC stage $\left(\mathbf{I C}_{P Q}\right)$. In each IC stage, the 


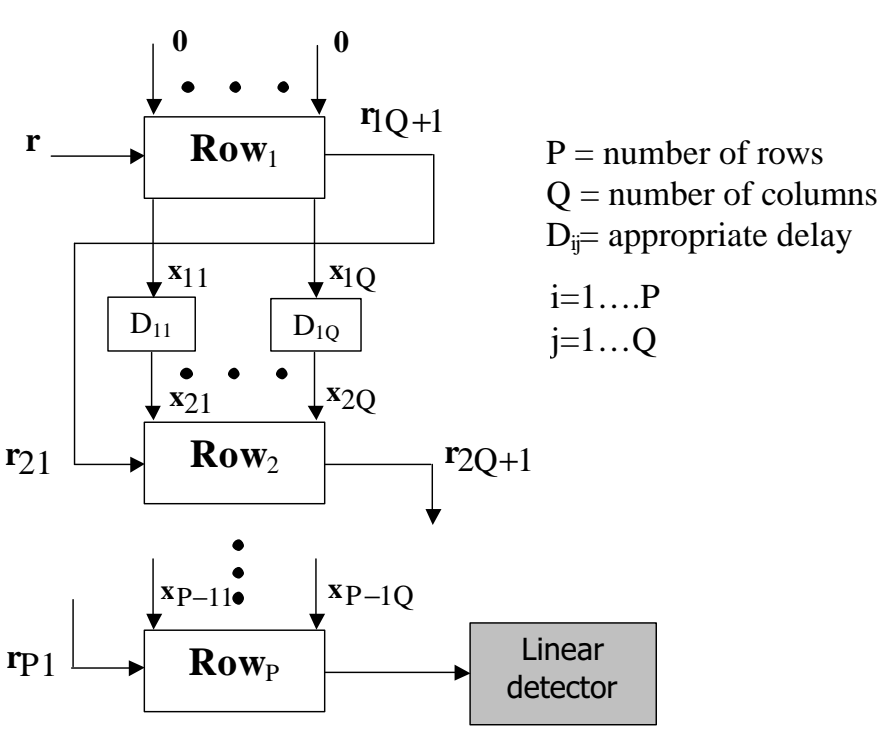

Figure 5. Vertical topology of the MS-NL-BIC receiver.

performance is measured after 1000 symbols used for the estimation in (14) and (18), and $F=5$ in (13). We assume the knowledge of the number of dominant interferers $Q$, which is the number of columns of the receiver matrix. Note that the issues related to the parameter $\mu$ (equation 7) are addressed later in this section.

For the case 1, Figure 7 depicts bit-error rate (BER) as a function of signal to background noise ratio (SNR) (with respect to the desired user). The results are obtained after a total of $P=4$ rows and $Q=7$ columns, which is where the BER reaches minimum. Additional IC stages do not introduce any improvement for this particular example. For $S N R=8 d B$, BER versus total number of IC stages is presented in Figure 8. Note that in this example the MS-NLBIC(MMSE) performs better than the MS-NL-BIC(MF). In this case, after the last IC stage ( $\left.\mathbf{I C}_{47}\right)$ MAI is still present, therefore, BMMSE detector can further improve the performance of the MS-NL-BIC receiver in the case 1.

Equivalent results, for the case 2, with $P=4$ and $Q=3$ and $S N R=8 d B$ are shown in Figures 9 and 10, respectively. In Figure 10, note that the MS-NL-BIC(MMSE) converges faster with respect to number of IC stages, but, at the end the MS-NL-BIC(MF) offers lower BER for this particular example. In this case, after the last IC stage $\left(\mathbf{I C}_{43}\right)$, MAI is almost completely removed. Introduction of the BMMSE as linear detector in the MS-NL-BIC receiver, may cause a drop in the performance due to estimation errors of the covariance matrix (in (18)) which is used to derive the BMMSE detector (this particular topic is analyzed in [8]).

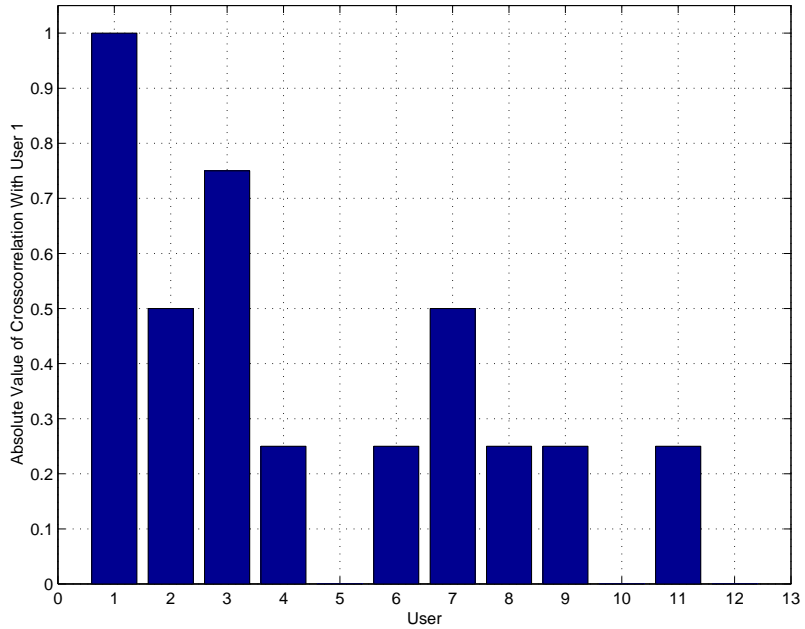

Figure 6. Absolute value of crosscorrelation between user 1 and user $\mathbf{i}(i=1, \cdots, 12)$.

We consider the performance of our receiver in the case 3 , which is an overloaded DS-CDMA system. Figure 11 depicts BER versus SNR (with respect to the desired user). The same figure presents the performance of the matched filter (denoted as MF-8) for the system without the strong interferers (only the desired user and eight equal-energy interferers, which is identical to ideal cancellation of the users 2 , 3 and 4). The receiver with $Q=7$ and $P=4$ is used. From the results in Figure 11, we note that the MS-NL-BIC completely cancels the strong users i.e, the MS-NL-BIC(MF) performance is identical to the MF-8 performance (in Figure 11 their characteristics overlap).

From these results, it is seen that the MS-NL-BIC outperforms linear receivers (MF and BMMSE), significantly. The performance of the linear receivers is expected because it is well known that they do not perform well in the case of the systems with strong and highly correlated interferers (with respect to desired user signature sequence) [12], as may be the case in overloaded systems. But, these results suggest that the MS-NL-BIC may be applied as a blind solution in the case of overloaded systems with strong interferers.

Let us now examine the effects of the choice of the parameter $\mu$ (equation 7) on the MS-NL-BIC scheme. For the case $1(S N R=4 d B, Q=7$ and $P=5)$ and the case $2(S N R=4 d B, Q=3$ and $P=5$ ), Figure 12 depicts BER of the desired user, as a function of $\mu$. In each IC stage, the MS-NL-BIC(MF) and the MS-NL-BIC(MMSE) apply same parameter $\mu$, which is presented on the abscissa. From these results, it is seen that the performance of the scheme is affected by the choice of the parameter $\mu$. We show that for different cases (i.e., system loading), different values of $\mu$ result in best performance. The issue of optimal design of 


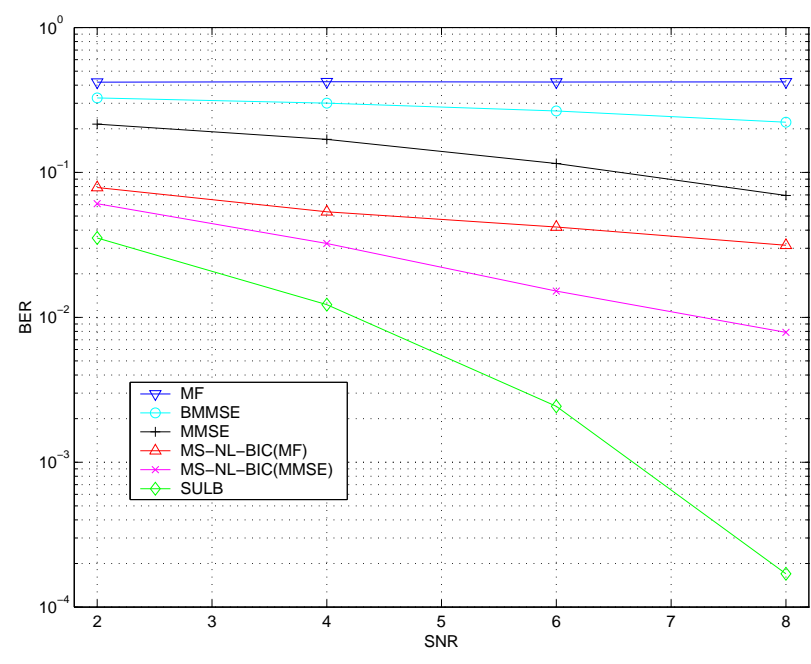

Figure 7. BER vs. SNR , $L=8, M=8$, $A_{i}^{2} / A_{1}^{2}=25, i=2, \cdots, 8$.

the parameter $\mu$ is a subject of future study.

\section{Conclusion}

We have introduced the MVE-MME optimization criterion which is then used to implement the MS-NL-BIC receiver. The receiver is based on determining the component of the received vector that has significant mean energy and low variability in the energy. The MS-NL-BIC consists of multiple IC stages, and can be viewed as a matrix of IC stages. The columns of the matrix resemble multistage receivers that iteratively refine performance from earlier stages, while each row corresponds to a blind equivalent to the nonlinear centralized SIC scheme. The ability of the receiver to exceed the performance of the linear receivers is confirmed via the simulation results. It is seen that this scheme is particularly effective for systems with very strong interferers which are strongly correlated with the desired user signature sequence. Therefore, this may be a very viable solution for implementation in the case of overloaded systems with strong interferers.

\section{References}

[1] A. Duel-Hallen. A Family of Multiuser Decision-Feedback Detectors for Asynchronous Code-Division Multiple-Access Channels. IEEE Transactions on Communications, 43:421434, Feb./March/April 1995.

[2] M. Honig, U. Madhow, and S. Verdú. Blind Adaptive Multiuser Detection. IEEE Transactions on Information Theory, 41(4):944-960, July 1995.

[3] R. Lupas and S. Verdú. Linear Multiuser Detectors for Synchronous Code-Division Multiple-Access Channels. IEEE

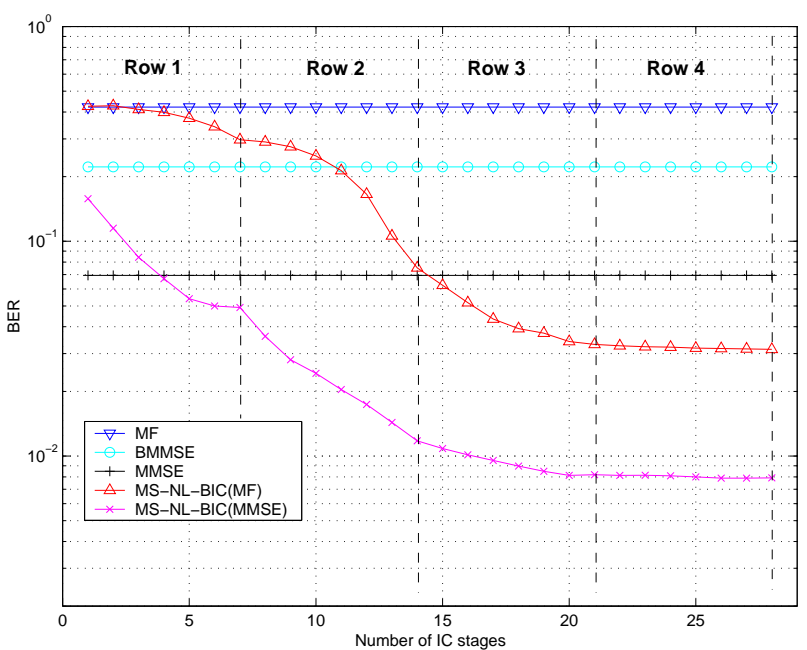

Figure 8. BER vs. number of IC stages, $L=8$, $M=8, A_{i}^{2} / A_{1}^{2}=25, i=2, \cdots, 8, S N R=8 d B$.

Transactions on Information Theory, 35:123-136, January 1989.

[4] U. Madhow. Blind Adaptive Interference Suppression for Direct-Sequence CDMA. IEEE Proceedings, Special Issue on Blind Identification and Equalization, pages 2049-2069, October 1998.

[5] U. Madhow and M. Honig. MMSE Interference Suppression for Direct-Sequence Spread-Spectrum CDMA. IEEE Transactions on Communications, 42(12):3178-3188, December 1994.

[6] P. Patel and J. Holtzman. Analysis of a Simple Successive Interference Cancellation Scheme in DS/CDMA Systems. IEEE JSAC, Special Issue on CDMA, 12(4):796-807, June 1994.

[7] B. Picinbono. Random Signals and Systems. Prentice Hall, 1993.

[8] D. Samardzija, N. Mandayam, and I. Seskar. Blind Interference Cancellation for the Downlink of CDMA Systems. Conference on Information Sciences and Systems, 2:TP3.17TP3.22, March 2000. Princeton University.

[9] I. Seskar, K. Pedersen, T. Kolding, and J. Holtzman. Implementation Aspects for Successive Interference Cancellation in DS/CDMA Systems. Wireless Networks, (4):447-452, 1998.

[10] Y. Z. Tsypkin. Adaptation and Learning in Automatic Systems. Academic Press, 1971.

[11] S. Ulukus and R. Yates. A Blind Adaptive Decorrelating Detector for CDMA Systems. IEEE JSAC, 16(8):1530-1541, October 1998.

[12] S. Verdú. Multiuser Detection. Cambridge University Press, 1998.

[13] X. Wang and V. Poor. Blind Multiuser Detection: A Subspace Approach. IEEE Transactions on Information Theory, 44(2):677-690, March 1998. 


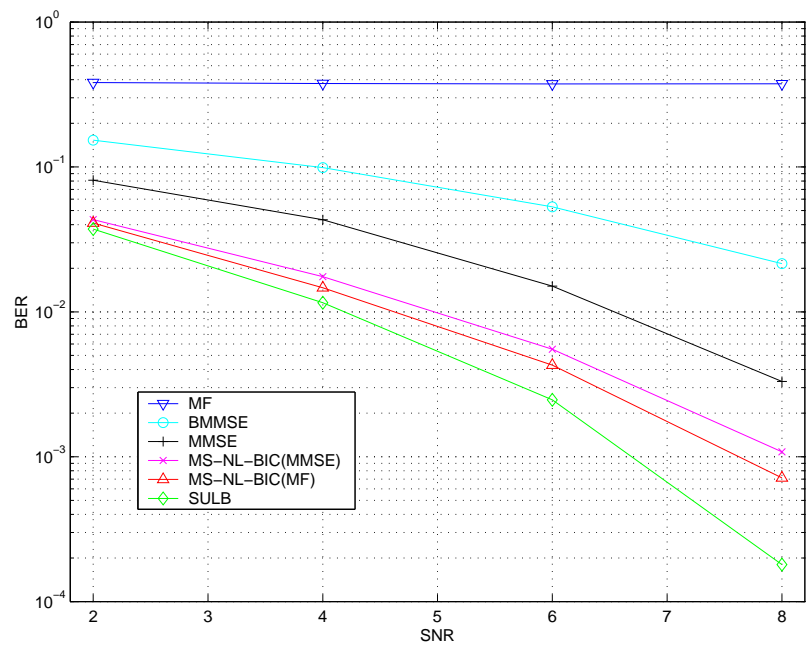

Figure 9. BER vs. SNR, $L=4, M=8, A_{i}^{2} / A_{1}^{2}=$ $25, i=2, \cdots, 4$.

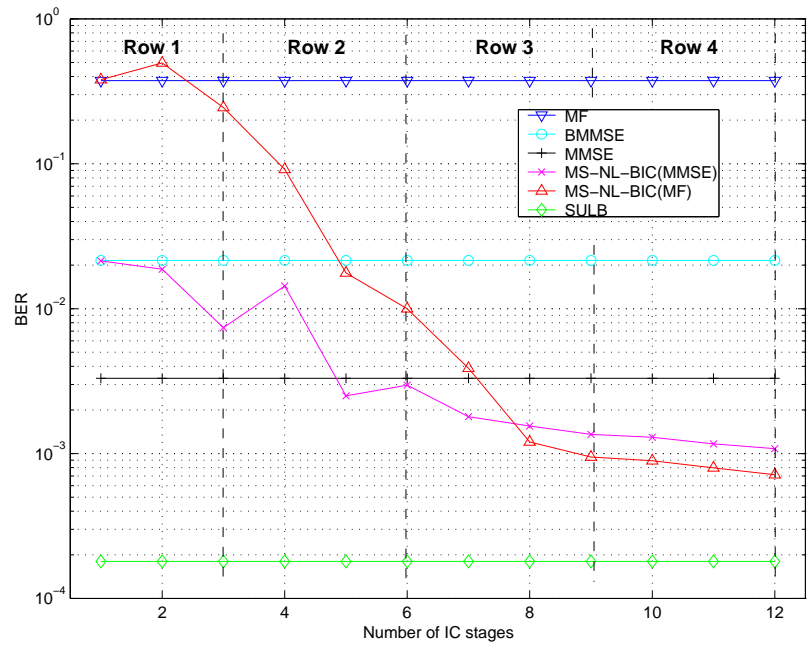

Figure 10. BER vs. number of IC stages, $L=$ $4, M=8, A_{i}^{2} / A_{1}^{2}=25, i=2, \cdots, 4, S N R=$ $8 d B$.

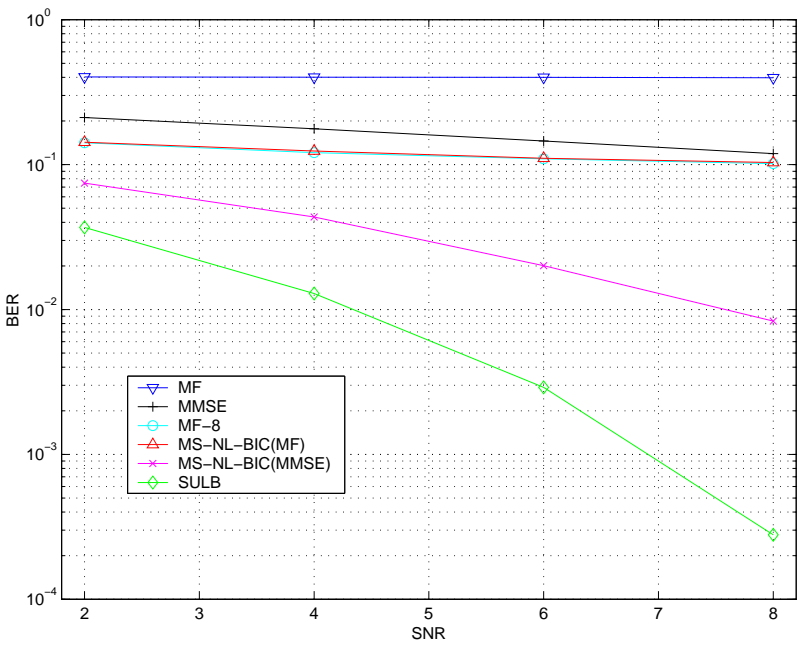

Figure 11. BER vs. SNR, $L=12, M=8$, $A_{i}^{2} / A_{1}^{2}=25, i=2, \cdots, 4$ and $A_{i}^{2} / A_{1}^{2}=1, i=$ $5, \cdots, 12$.

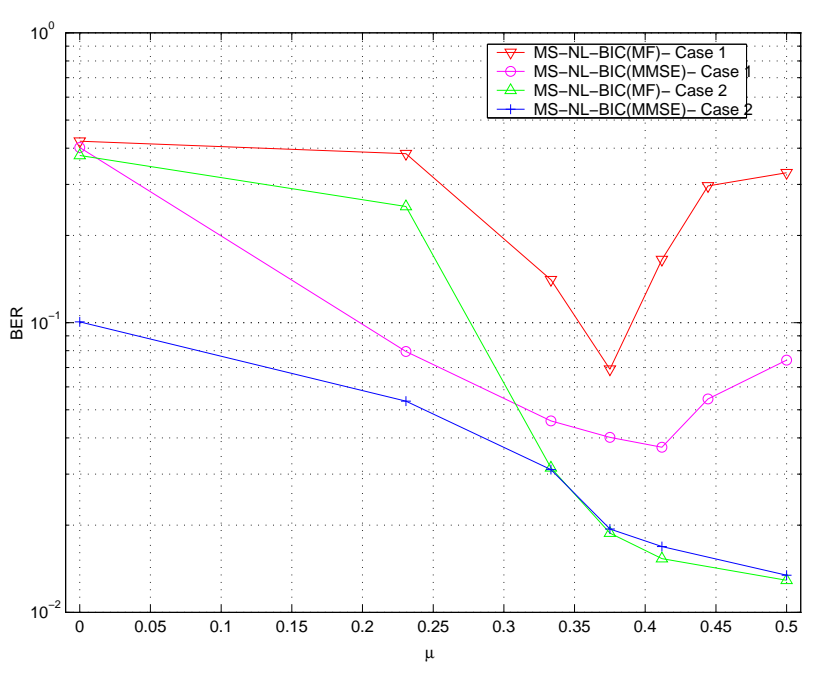

Figure 12. BER vs. parameter $\mu$, Case 1 and 2, $S N R=4 d B$. 